\title{
LA REORDENACIÓN DEL SISTEMA DE TRANSPORTE PÚBLICO ANTE EVENTOS ESPECIALES, UN EJERCICIO PRÁCTICO DE COORDINACIÓN INTERMODAL
}

\author{
Pedro Galindo González \\ Nuria Blanco Caballero \\ Carmen Moreno Muñoz \\ Alejandro Chico González
}

Consorcio Regional de Transportes de Madrid

\section{RESUMEN}

El Consorcio Regional de Transportes de Madrid (CRTM), como autoridad de transporte público de la región, coordina los más de 40 operadores públicos y privados para dar una respuesta óptima a las necesidades de movilidad de los ciudadanos.

El transporte público en la Comunidad de Madrid constituye un sistema complejo de carácter intermodal, en el que operan modos de transporte urbanos e interurbanos, conectados mediante un conjunto de intercambiadores o áreas intermodales que canalizan la movilidad radial entre la corona metropolitana y la capital.

En este contexto, el CRTM ha puesto en servicio su Centro de Innovación y Gestión del Transporte Público, CITRAM, que representa un nuevo paso en la integración del sistema, modificando el papel de la autoridad de transporte, que pasa de ser un mero observador del sistema a un componente activo del mismo. La misión de CITRAM es proporcionar servicios de información integrada, conectividad y cooperación entre todos los agentes implicados en el sistema de transporte público de la región.

Es una prioridad para el CRTM estar preparado para la gestión multimodal ante eventos especiales que puedan suponer una reordenación del sistema de transporte: grandes afecciones en superficie que afecten a las redes de autobuses, restricciones de tráfico por episodios de contaminación atmosférica, cortes de metro por obras, etc..

La gestión de este tipo de situaciones exige un nivel de coordinación e información muy complejo, que implica una planificación adecuada de las alternativas de transporte de los usuarios, así como la coordinación de todos los agentes involucrados (autoridad de transporte público, agencias de gestión de tráfico, operadores de transporte, servicios de emergencias, etc.). Además, a través del uso de TICs, la implantación dinámica de las acciones coordinadas es más ágil y flexible y se aumenta la capacidad de reacción para su despliegue. 


\section{INTRODUCCIÓN}

En la ciudad de Madrid es cada vez más habitual la convocatoria de grandes eventos, que ponen a prueba la capacidad del sistema de transportes de la región: competiciones deportivas, manifestaciones, grandes concentraciones, etc. En general, en este tipo de eventos multitudinarios, el sistema de transporte ocupa un papel determinante para garantizar la movilidad simultánea de participantes y usuarios habituales, adaptando la oferta a un escenario completamente nuevo con necesidades singulares a atender.

Del mismo modo, también son recurrentes otro tipo de circunstancias especiales, intrínsecas o no al sistema de transportes, que modifican sustancialmente la configuración urbana y los esquemas de movilidad de la población, como pueden ser las nevadas en determinadas zonas de la sierra, las restricciones al vehículo privado en determinadas zonas de la ciudad en horas del día, obras, etc.

Ambas circunstancias, sin ser "idénticas", son similares, pues exigen un ejercicio de reordenación del sistema y adecuar la oferta de los modos de transporte en su conjunto para poder canalizar la demanda afectada dentro del sistema y atender de manera eficaz los incrementos de demanda que pudieran producirse.

Por otro lado, es necesario tener muy presente las demandas de información de movilidad por parte de los ciudadanos. Actualmente, el sistema de transporte público de la región cuenta con más de 5 millones de viajeros al día. La mayoría de estos viajeros son usuarios multimodales que demandan información dinámica, combinada y de calidad sobre el mismo para optimizar sus viajes ante cualquier tipo de circunstancia que suceda en el sistema de transporte o que pueda afectar a la misma.

Para dar respuesta a todos los eventos que suceden en la Comunidad de Madrid (sólo durante el año 2015 se analizaron más de 1000 eventos con repercusión, mayor o menor, sobre el sistema de transportes) y a la creciente demanda de los viajeros, el Consorcio Regional de Transportes de Madrid (CRTM) como autoridad de transporte público de la región, lleva años trabajando en la planificación de las actuaciones ante grandes eventos especiales.

Además, la gestión dinámica en tiempo real de esta planificación es más ágil y eficaz gracias a la existencia de CITRAM, el Centro de Innovación y Gestión del Transporte Público del CRTM, que permite la integración de información en tiempo real de las infraestructuras y servicios de los diferentes modos de transporte que operan en la Comunidad de Madrid, apoyándose en la innovación tecnológica, para una mejor toma de decisiones, resultando en una gestión efectiva del sistema de transporte público en tiempo real. 


\section{PLANIFICACIÓN DE TRANSPORTE PÚBLICO ANTE EVENTOS ESPECIALES}

La planificación de las actuaciones y el seguimiento de las mismas requieren una visión de conjunto y una coordinación de todos los modos y operadores que forman el sistema de transporte de la Comunidad de Madrid, función que compete al CRTM, para poder aprovechar las sinergias de un sistema integrado, intentando adecuar las necesidades de transporte a la oferta disponible.

En este apartado se exponen los principales elementos que suelen tenerse en cuenta a la hora de plantear la reordenación del transporte cuando surgen eventos o circunstancias que requieren un esfuerzo de las infraestructuras y servicios por encima de su capacidad en condiciones normales.

\subsection{Tipo de evento}

En primer lugar, es esencial conocer las características del evento al que nos estamos enfrentando, ya que la planificación de la operativa alternativa depende de múltiples factores a considerar en el análisis (finalidad, afluencia de personas, duración, perfil de usuario o tipo de público, etc.).

- Es necesario conocer la finalidad del evento, puesto que las afecciones y necesidades varían según la naturaleza del mismo; así encontramos que las concentraciones de público pueden localizarse en un ámbito determinado o estar en movimiento ("marchas”), conllevar mayores niveles de restricción a peatones, viajeros y tráfico, según niveles de riesgo en situaciones de emergencia, alterar en mayor o menor medida los patrones de movilidad en el caso de cortes por mantenimiento y renovación de infraestructuras (tramos viarios, tramos ferroviarios, etc..).

- Por otro lado, dos eventos de la misma naturaleza pueden tener una escala diferente; así, pese a tener características comunes, no se tratan de la misma forma eventos multitudinarios frente a eventos más locales, grandes procesiones que pequeñas manifestaciones, el cierre de una línea completa de metro o el corte de un tramo intermedio,...

- Además, resulta fundamental delimitar el momento temporal en el que se va a producir el evento para poder estimar la demanda afectada y dimensionar la oferta, lo que incluye analizar los horarios, la duración y el período en el que se produce (eventos diurnos o nocturnos, en laborable o en fines de semana, en temporada de invierno o temporada baja de verano). En este sentido, cobra especial relevancia el grado de incertidumbre del evento, ya que los eventos pueden suceder de manera prevista o ser imprevistos (pese a disponer de protocolos de actuación), como 
puede ser el caso de la suspensión de servicio en un tramo de Cercanías por obras o para resolver una incidencia puntual.

- Finalmente, otro aspecto relevante a la hora de planificar un evento es la localización del mismo, ya que dependiendo de su ubicación en el centro urbano, en zonas suburbanas, en el interior de barrios,... el nivel de afección y la densidad de las redes de transporte determinarán el tipo de solución a adoptar (refuerzo de oferta, servicios especiales, cierre de accesos a estaciones, etc.)

A partir del análisis de estos elementos se podrán acotar y determinar los datos de partida necesarios para la planificación del dispositivo de movilidad, en la cual, independientemente del tipo de evento a gestionar, una de las claves fundamentales consiste en integrar, en la medida de lo posible, la gestión operativa del transporte y la del propio evento de manera coordinada.

\subsection{Diagnóstico y criterios de actuación.}

Para definir las acciones de transporte público a implantar, conocida la tipología del evento, es necesario analizar la situación existente en el ámbito para valorar el posible nivel de afección sobre las redes de transporte (oferta, demanda, instalaciones,...). A partir del resultado del contraste entre la ordenación actual y el nuevo escenario de movilidad previsto, se podrá redimensionar la oferta de manera eficaz y plantear propuestas específicas de reordenación que aprovechen la intermodalidad del sistema.

\section{- Localización y delimitación del área afectada}

Se trata de reproducir el área urbana o suburbana afectada por el evento que se va a producir: recinto cerrado, calle por donde discurre una maratón, ámbito afectado por el corte de una canalización de agua, vías cerradas al tráfico, etc.

El paso siguiente consiste en definir, en la medida de lo posible, los condicionantes de acceso al ámbito, en cuanto a movilidad peatonal - flujos, accesos a paradas y estaciones (sin servicio, modificadas para sólo permitir entrada o salida, etc...)-, restricciones al vehículo privado, a las redes de transporte de superficie, a otros servicios, etc...

Con esta base de partida se tomarán decisiones que afectan a los cortes de tráfico, funcionamiento de estaciones y desvíos de líneas afectadas. En este punto es imprescindible contar con los agentes implicados, que ayudarán a definir con mayor precisión las características de la zona de afección, que se concreta en la figura del plan específico de movilidad. 


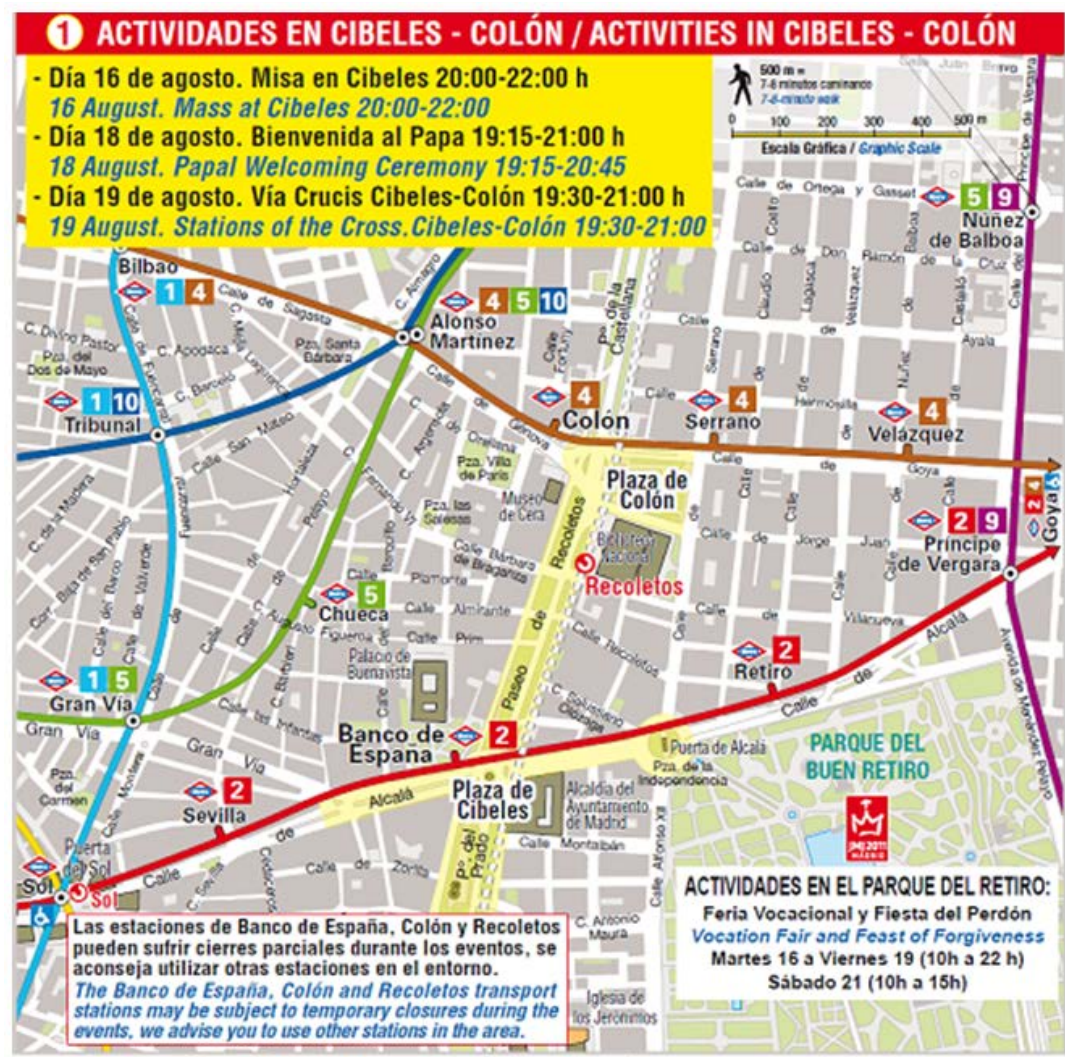

Fig. 1 - Accesibilidad en TP actos en Cibeles-Colón durante la JMJ (Madrid 2011)

\section{- Análisis de los modos afectados}

Definida la zona de actuación y las características del evento, se evalúa, en primer lugar, el impacto sobre el sistema de transportes para identificar tanto las redes, directa o indirectamente afectados, como los elementos del mismo que pudieran quedar sin servicio, o por el contrario, sometidos a cargas excesivas por encima de su capacidad teórica planificada (líneas, estaciones, paradas, etc...).

En función de este criterio se determinan las áreas sin servicio de transporte público y las áreas en las que el servicio pudiera ser insuficiente en función del contraste ofertademanda, así como los modos complementarios ya existentes que pudieran servir de apoyo a los directamente afectados.

En general, cualquier tipo de evento supone afección directa a alguno o varios de los modos, pero hay que tener en cuenta que el resto de modos se ven afectados indirectamente por la demanda derivada o generada como efecto de la propia reordenación del sistema de transportes.

\section{- Análisis de la oferta del sistema de transportes}

Con el objetivo de conocer la capacidad de los modos afectados, se realiza una evaluación de la oferta existente, en lo que respecta tanto a capacidad de transporte (pasos-hora, capacidad máxima, etc.), como a la capacidad de las propias infraestructuras (capacidad de 
vestíbulos, andenes y zonas de circulación en estaciones e intercambiadores, zonas de espera en paradas de autobús, espacios de regulación, etc.).

Como se ha indicado, en esta fase cobra especial importancia el período en que se produce el evento (hora punta/hora valle, diurno/nocturno, laborable/fin de semana, verano/invierno). Una vez que obtenemos la capacidad de los modos en el período en que se va a producir el evento, disponemos de la base de partida para, junto con el posterior estudio de demanda, conocer si nuestro sistema está preparado para hacer frente a la solicitación extra que supone el evento especial.

\section{- Análisis de la demanda de usuarios}

Finalmente, se realiza una valoración de la demanda estimada en el nuevo escenario y su adecuación a la oferta existente para determinar la propuesta de actuaciones a desarrollar.

Para este tipo de situaciones hay que considerar, principalmente, dos criterios para el análisis de demanda: por una parte, la demanda actual de viajeros en los modos de transporte afectados y, por otra, la demanda generada o atraída por el evento en cuestión. En el segundo caso, existe un grado de incertidumbre que se puede estimar en mayor o menor medida en función de la naturaleza y del nivel de definición que dispongamos del evento.

Existen otros factores a tener en cuenta en la caracterización de la demanda como son la duración del evento, el tipo de usuario, el sistema tarifario, la cautividad de los viajeros, etc.

\section{- Agentes implicados en la planificación.}

Paralelamente a estos análisis, una vez estudiada la tipología del evento, se determinarán los agentes implicados en el mismo. Teniendo en cuenta la relevancia del transporte público en toda la gama de eventos especiales descritos, resulta positivo que los distintos agentes implicados en la puesta en marcha del mismo participen en la planificación, de manera que se defina un plan de actuación conjunto y coordinado.

Estos agentes son, en función de la naturaleza del evento, los operadores de transporte, los servicios de emergencia y otros organismos como Ayuntamientos o DGT, y en determinados casos, la propia organización del evento. La coordinación con los agentes implicados se centraliza a través de CITRAM.

\subsection{Planteamiento de alternativas}

Partiendo del diagnóstico de la situación existente en la zona donde se va a localizar el evento, y realizada la valoración de la adecuación entre oferta y demanda, se determinarán las acciones específicas que respondan a criterios de optimización de redes 
(intermodalidad), y reducción de los problemas de accesibilidad y conectividad que pudiera causar la afección.

Como es habitual, en el diseño de redes de transporte las características propias de los distintos modos condicionan las soluciones a adoptar, en cuanto a capacidad, flexibilidad, velocidad comercial, longitud de itinerario, etc...adoptando como criterio de actuación potenciar la función de intercambio entre los distintos modos de transporte público, y aprovechando la flexibilidad de los modos de transporte en superficie para canalizar la demanda a los modos de alta capacidad.

Por tanto, de forma general, las acciones a implantar suelen ser las siguientes:

- Redes de autobuses: desvío de itinerarios, creación de servicios especiales, refuerzo de dotación de líneas (de cocheras o procedente de otras líneas con menor demanda), etc.

- Redes ferroviarias: Refuerzo de trenes (número y composición), servicios pasantes en determinadas estaciones, modificación de estado de funcionamiento de estaciones (cierre de accesos, control de flujos, refuerzo de personal).

Además de estas medidas, sobre la infraestructura viaria se deben tomar otras con el objetivo de adecuar la red a las modificaciones planteadas: análisis y posicionamiento de nuevas paradas, acondicionamiento de cabeceras, acceso a estaciones, cortes de tráfico, etc.

Otros factores que hay que tener en cuenta en el planteamiento de la reordenación son: compatibilizar las acciones con los viarios de acceso a la zona, evitar en la medida de lo posible zonas y períodos con congestión circulatoria, delimitar zonas con espacio suficiente para la regulación de autobuses, proporcionar seguridad e información suficiente al usuario (Apps, redes sociales, señalización específica, planos, entre otros).

\section{EL USO DE LAS TECNOLOGÍAS DE LA INFORMACIÓN Y LA COMUNICACIÓN (TICs) ANTE EVENTOS ESPECIALES}

Para la puesta en marcha de todas las acciones establecidas durante la planificación y para conseguir una gestión en tiempo real más eficaz ante cualquier evento que suponga una reordenación del sistema de transportes, CITRAM se apoya en las TICs para la gestión de los mismos. 


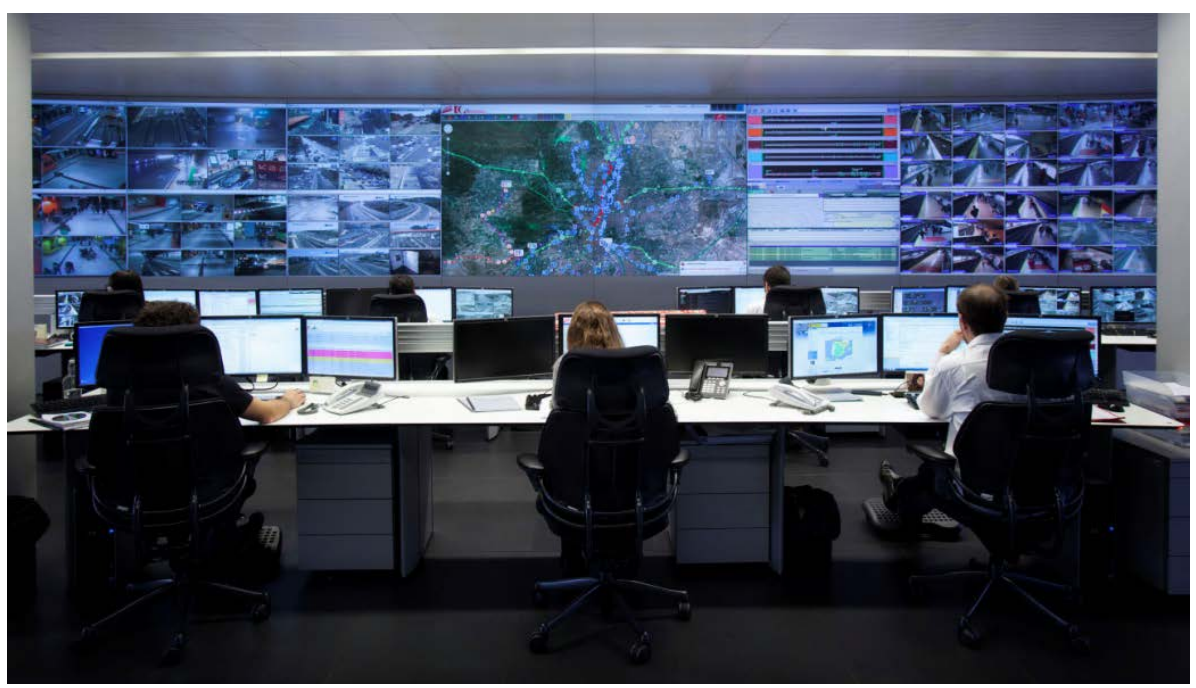

Fig. 2 - CITRAM, Centro de Innovación y Gestión del Transporte Público

El CRTM ha desarrollado herramientas multimodales "a medida" que integran información de los más de 40 operadores de transporte público que conforman el sistema integrado en una misma plataforma y permiten actuar con un enfoque global de sistema. Esto supone la visualización de más de 20.000 cámaras y la localización en tiempo real de más de 5.000 vehículos y 6.000 paneles de información variable. Además estas herramientas cuentan con información de la Dirección General de Tráfico (DGT), el 112 y el Ayuntamiento de Madrid, entre otros.

El objetivo primordial de las mismas es mejorar la rapidez en la detección de incidentes, valoración de su impacto y toma de decisiones asociadas por parte de los operadores de CITRAM. De igual modo, estas herramientas hacen posible la provisión de información a los usuarios de manera rápida y efectiva. Entre estas herramientas cabe destacar las siguientes:

- Módulo de alertas tempranas: permite la identificación y generación automática de alarmas ante posibles circunstancias que afecten al sistema de transporte público;

- Motor de decisión: herramienta que permite la gestión más rápida y eficiente de los incidentes y eventos gracias a la propuesta automática de acciones que ayudan a los operadores en la toma de decisiones y el seguimiento de los incidentes;

- Módulo de distribución de información (MDI): permite el envío de información adaptada a las circunstancias de cada perfil a través de múltiples canales de envío de información;

- Supervisor Gráfico: aplicación que permite localizar en un mapa en tiempo real la posición de más de 5.000 vehículos del sistema de transporte público (STP), ilustrando gráficamente recorridos de líneas, incidencias, etc.;.

- CCTV multimodal: aplicación que integra sistemas de CCTV de toda la red de transporte de la región (estaciones de Metro, intercambiadores, metros ligeros, cámaras de tráfico, embarcadas...), en total más de 20.000 cámaras; 
- Gestor de Incidencias (GEIS): plataforma web en la que se registran incidentes y afecciones al servicio por parte de los operadores de transporte;

- Sistema de Gestión de Intercambiadores: Permite conocer el estado de las instalaciones en tiempo real, gestionar alarmas, seguimiento de tráfico y ocupación de dársenas, mantenimiento, etc. de todos los intercambiadores de la Comunidad de Madrid;

- Sistema de información al usuario: herramienta a través de la que se emite información en tiempo real en los paneles de información al viajero de los intercambiadores. También se utiliza para emitir información en tiempo real a bordo de los autobuses interurbanos en servicio en ese momento.

Una vez que CITRAM recibe la planificación de la reordenación del STP ante el evento especial, se siguen una serie de acciones con el objetivo de coordinar a los agentes implicados, con el fin último de homogeneizar la información en tiempo real al usuario, de tal forma que pueda planificar su viaje. Para ello, se diferencian, por un lado, las acciones previas al evento especial y, por otro, las realizadas durante el mismo.

\subsection{Actuaciones previas al evento}

Recibida la planificación, el CRTM, a través de CITRAM, se pone en contacto con todos los agentes implicados para la preparación previa al evento especial.

Generalmente intervienen:

- CRTM/CITRAM

- Gestores de infraestructuras y Operadores de transporte:

o Intercambiadores

o Metro/Metro Ligero

o EMT (autobuses urbanos de Madrid)

o Autobuses interurbanos

o Renfe Cercanías

o Autobuses urbanos de otros municipios

o Largo recorrido

- Servicios de emergencia:

o 112

o CISEM (Centro Integrado de Seguridad y Emergencias de Madrid)

o Policía municipal

o Bomberos

o SAMUR - Protección Civil

o Policía Nacional

o Guardia Civil 
- Otros organismos:

o Centro de pantallas del Ayuntamiento de Madrid (Área de Movilidad)

o DGT

o Ayuntamientos de otros municipios

Debido a la magnitud de estos eventos se realizarán comunicaciones previas al mismo de cara a asegurar que todos los agentes tengan claro las acciones que deben realizar a lo largo del mismo y las herramientas y canales de comunicación a emplear.

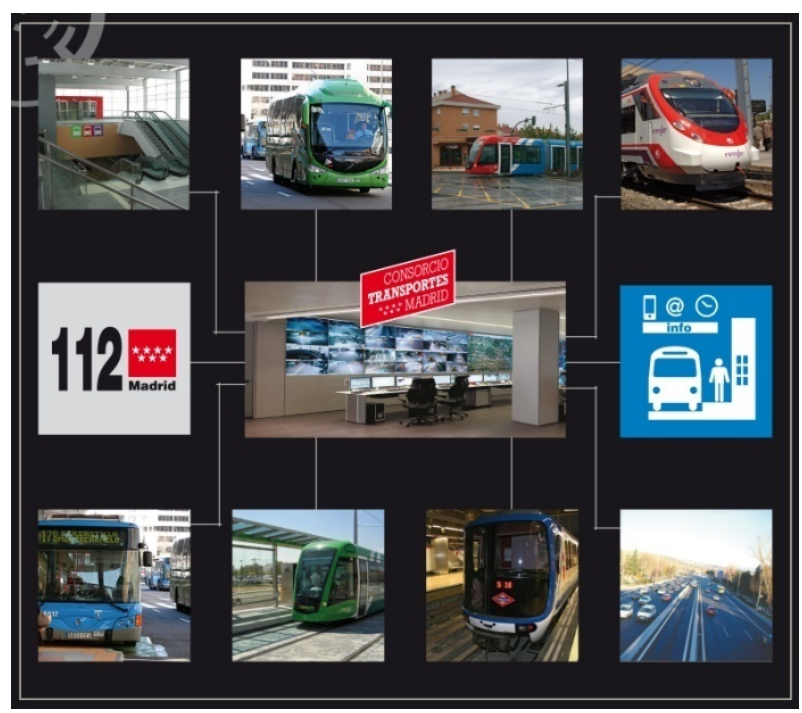

Fig. 3 - Agentes implicados

Además, desde CITRAM, siempre que sea posible, se informa al usuario sobre el evento especial para que sea consciente de las posibles repercusiones que puede tener el mismo sobre sus pautas de movilidad diarias. Esta información se envía a todos los canales disponibles para ello (pantallas en intercambiadores, teleindicadores en paradas de autobuses urbanos e interurbanos, a bordo en interurbanos, en la App Mi Transporte, en la página web del CRTM....).

Se hace también un seguimiento del grado de difusión del evento, y el posible impacto del mismo, a través de redes sociales, de cara a analizar el grado de conocimiento de las medidas ante el evento especial, primeras impresiones, reacciones y comentarios de los usuarios.

Por último, de cara a los organismos que pueden verse involucrados $\mathrm{y}$ a las comunicaciones a realizar en importante tener clara la estructura organizativa ante el evento. Esta variará según el tipo de evento, pero desde un punto de vista genérico puede conformarse del siguiente modo: 
- Delegación de Gobierno

- Consejería de Transportes, Vivienda e Infraestructuras de la Comunidad de Madrid

- Ayuntamiento de Madrid

- CRTM

\subsection{Actuaciones a realizar durante el desarrollo del evento}

Una vez comience el evento,

el primer hito para una correcta gestión del mismo es la detección temprana de cualquier incidente o circunstancia que se salga de lo planificado y que pueda tener afección sobre el transporte público. Para ello, se ha creado una herramienta, el módulo de alertas tempranas, que basada en una serie de reglas predefinidas y gracias a un conjunto de indicadores provenientes de diversas fuentes (localización de vehículos en tiempo real, obras, niveles de circulación, alertas sobre contaminación, información de los servicios de emergencias...) es capaz de identificar cualquier circunstancia que pueda afectar al correcto funcionamiento del sistema de transporte público. Cuando esto ocurre el módulo de alertas tempranas lanza un aviso a los responsables de sala de CITRAM que serán los encargados de coordinar las acciones posteriores.

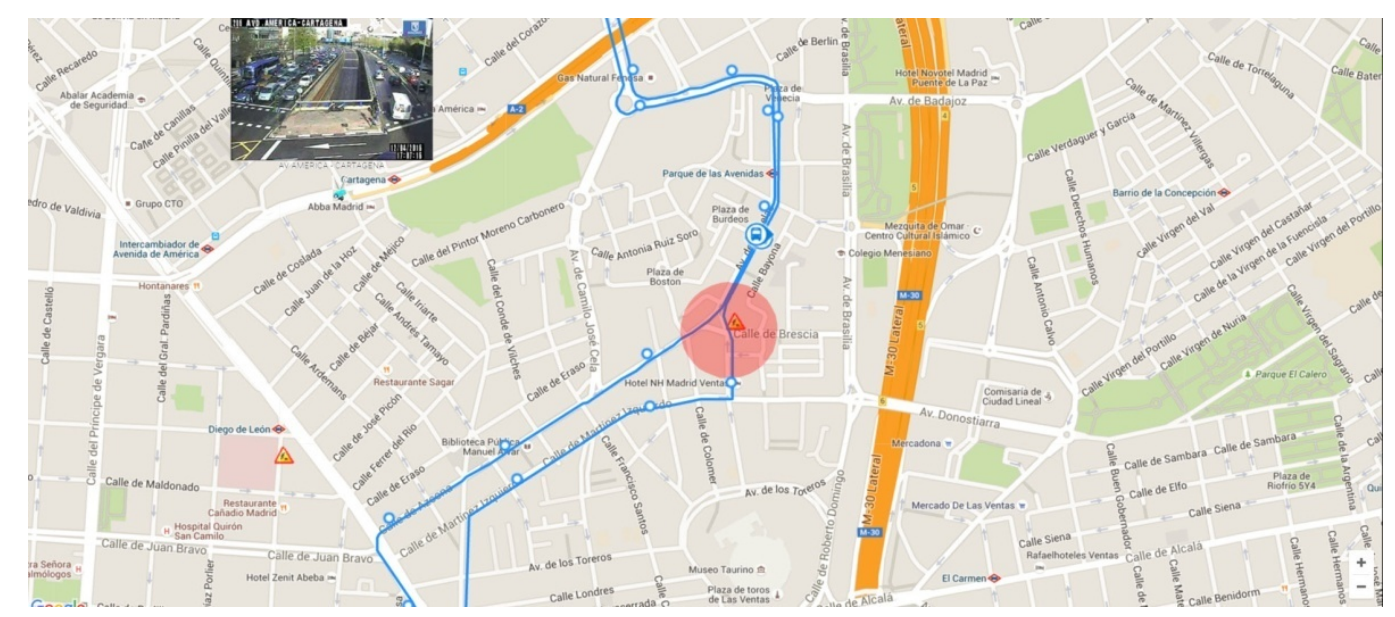

Fig. 4 - Interfaz del módulo de alertas tempranas

En este contexto, en función de la magnitud y relevancia del evento, es habitual constituir gabinetes de crisis en el CRTM, de modo que, tanto los servicios técnicos del Organismo como representantes de los operadores y miembros de los Cuerpos y Fuerzas de Seguridad, en su caso, evalúan el desarrollo de la acción y coordinan las medidas correspondientes, de manera centralizada.

El módulo de alertas tempranas está ligado a otro sistema, imprescindible para la gestión coordinada ante grandes eventos, el motor de decisión. Esta herramienta es un asistente gráfico cuyo objetivo primordial es servir de apoyo a los operadores de CITRAM en el seguimiento de la afección, facilitando la toma de decisiones y proponiendo soluciones y 
acciones ante ciertas circunstancias que se aparte de lo previamente planificado. Gracias a la integración de información geográfica de todo el territorio y a la existencia de una base de datos de cámaras y paneles de información de transporte de toda la región, el motor de decisión es capaz de calcular en tiempo real y de forma dinámica el área de influencia y la red de transporte influenciada por el evento, qué agentes se verán implicados en el mismo (servicios de emergencia, operadores de transporte, usuarios...), qué cámaras son las más adecuadas para el seguimiento del mismo y la identificación de los paneles de los distintos modos de transporte relevantes para el envío de información a los usuarios durante el desarrollo del evento.

Para el envío de información, no sólo a los usuarios del transporte sino también a cualquier agente que se pueda beneficiar de la misma (operadores de transporte público, personal en campo, servicios de emergencia..) se ha creado el módulo de distribución de información (MDI). Basada en una serie de escenarios predefinidos y otra información introducida directamente en el sistema o ligada a la del motor de decisión, mediante grupos de perfiles y zonas de distribución, el MDI es capaz de enviar mensajes predefinidos, dependiendo de las circunstancias, a través de los diferentes canales integrados en él (paneles de información a los usuarios de los diferentes modos de transporte e infraestructuras, página web del CRTM, aplicaciones móviles desarrolladas, mensajes de texto a teléfonos móviles y emails, entre otros).

\section{CONCLUSIONES}

Madrid es una región en la que son muchos los eventos de todo tipo que se suceden a lo largo del año, y la sociedad demanda cada vez más información, más precisa y de manera inmediata.

Se ha visto que el primer paso es planificar para el nuevo escenario, con el objetivo de reordenar el sistema de transporte público, optimizando la capacidad del mismo, de tal forma que se consiga adaptar la oferta a la nueva situación de la demanda.

Para que dicha planificación surta un efecto positivo, es necesario coordinar a todos los operadores de transporte, informar de manera dinámica a los clientes del transporte público y supervisar los efectos y las desviaciones de las acciones planificadas.

El objetivo último es que la información relevante llegue homogénea al ciudadano, y pueda realizar su viaje con garantías de éxito.

El Consorcio Regional de Transportes de Madrid tiene garantizadas esta supervisión e información continua gracias a CITRAM, Centro de Innovación y Gestión del Transporte Público de Madrid, centro 24/7 desde el que se gestiona de una forma integrada todo el transporte público de la Comunidad de Madrid en tiempo real. 